\title{
The TROIKA beamline at ESRF
}

\author{
G. Grübel, J. Als-Nielsen(1) and A.K. Freund \\ European Synchrotron Radiation Facility, BP. 220, 38043 Grenoble, France
}

\begin{abstract}
The Troika beamline is a high-brilliance multi-station undulator beamline that operates with $\mathrm{x}$-ray transparent diamond and/or beryllium monochromators. A series of such transmission monochromators can use the same white beam and supply several experimental stations simultaneously with monochromatic x-rays of slightly different energies (beam-multiplexing). The favorable thermal and crystallographic properties of perfect diamond are well matched to the characteristics of the new low emittance undulator sources. The low absorption of both diamond and beryllium make beam-multiplexing to an attractive alternative to conventional single-station beamlines. The Troika beamline operates between $6 \mathrm{keV}$ and $18 \mathrm{keV}$ taking advantage of the gap tunable undulator. First experiments exploring the brightness, polarization properties and the high flux of coherent $\mathrm{x}$-rays produced reveal the superior potential of third generation synchrotron sources.
\end{abstract}

\section{The TROIKA Concept}

The TROIKA project is concerned with the operation of a multi-station undulator beamline at the European Synchrotron Radiation Facility (ESRF). The simultaneous operation of several experimental stations (beam-multiplexing) is achieved by using low $Z$, $x$-ray transparent diamond and/or beryllium transmission monochromators, that allow $x$-rays which are not diffracted by the first monochromator to be transmitted into downstream monochromators and their attached experimental stations. The principle of a multi-station beamline is sketched in Figure 1. The undulator source produces radiation at harmonics $\left(n * E_{0}\right)$ of the fundamental energy $E_{0}$ with a relative bandwidth $\Delta \lambda / \lambda \approx 1 / \mathrm{nN}=10^{-2}$ for the third harmonic of an undulator with period $N=36$. The first monochromator is set to reflect $x$-rays of energy $E_{1}$ for the first experimental station. The relative bandwidth extracted from a white undulator beam by a thin perfect diamond monochromator is typically $10^{-4}$ and therefore small compared to an undulator harmonic with a bandwidth of $10^{-2}$. This is illustrated in the (middle) insert of Figure 1, showing the transmitted spectrum of an undulator harmonic. Apart from the narrow intensity dip (50\% for a thick crystal in symmetric Laue geometry) caused by the first monochromator (diffracting at energy $E_{1}$ ) and other possible, but weaker Bragg reflections the transmitted $\mathrm{x}$-ray beam is practically not attenuated or perturbed. A second transmission monochromator set to reflect photons of a slightly different energy $\mathrm{E}_{2}$ can supply a second station with monochromatic $x$-rays. The transmitted beam shows now 2 main dips at energies $E_{1}$ and $E_{2}$ and can be used by further down-stream stations equipped with transparent monochromators or by an endstation operating with a conventional monochromator.

Transmission monochromators not only multiply the use of an undulator source but also provide a natural solution for the heatload problems encountered at third generation insertion device sources, because the thermal properties of both diamond and beryllium are superior to those of the classical Si and Ge crystals. Table 1 compares the relevant parameters for the new transmission monochromators with the characteristics of conventional monochromator crystals. Diamond and beryllium have a small absorption coefficient, a low coefficient of thermal expansion and a high thermal conductivity. The figure of merit $\kappa / \mu \alpha$ is 259 and 0.45 for diamond and silicon, respectively. Perfect diamond single crystals provide a better intrinsic energy resolution $\Delta \lambda \lambda$ than Si monochromators and their angular acceptance $\Delta \lambda \lambda \approx \tan \theta$ is well matched to the new low emittance synchrotron undulator radiation sources. Values for the intrinsic energy resolutions $\Delta \lambda / \lambda$, calculated for the symmetrical Bragg case and linear in-plane $(\pi)$ polarization are

(1) Permanent address: Riso National Laboratory, Roskilde, Denmark 


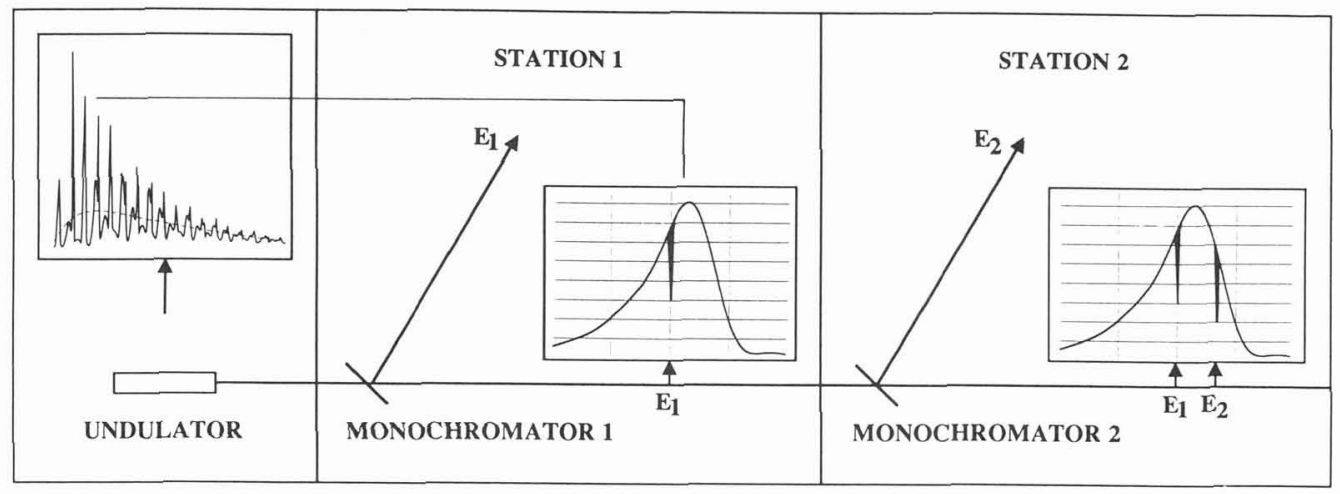

Figure 1: Schematic layout of a multiplexing beamline with two x-ray transparent monochromators. The transparent monochromators ( 1 and 2 ) cause narrow intensity dips in the transmitted spectra (see text).

also given in Table 1. Perfect diamond is available at sizes big enough $\left(8 * 8 \mathrm{~mm}^{2}\right)$ to operate a multi-station beamline even without focussing devices if the undulator is placed in a high- $\beta$ section (typical rms divergences $<20 \mu \mathrm{rad}$ ) of the storage-ring. Mosaic beryllium crystals (mosaic distribution typically 200 $\mu \mathrm{rad}$ ) have less favorable thermal properties but are five times as transparent as diamond. Due to their bigger bandwidth (typically $5 * 10^{-4}$ for the $(002)$ reflection), they are well suited for experiments that require maximum flux and can afford a slightly degraded divergence [1].

The Troika concept was approved for the open undulator beamline (BL9) at ESRF and will be implemented in two steps. Phase I, a single-station beamline (TROIKA I) with one transparent diamond monochromator, is completed. TROIKA I allows already the use of the white, transmitted beam and supports a second monochromator for test purposes. In a later phase the full TROIKA concept will be implemented comprising two $\mathrm{x}$-ray transparent monochromators and presumably a double-crystal fixed-exit monochromator for the TROIKA end-station. In this phase up to 3 experimental stations will operate simultaneously.

\section{The TROIKA I Beamline}

A schematic layout of the TROIKA I beamline is shown in Figure 2. The frontend (FE), located $23 \mathrm{~m}$ from the insertion device (ID) interfaces the beamline to the storage ring and contains the main beam shutter, a beryllium window $(500 \mu \mathrm{m})$ and Carbon filters $(252 \mu \mathrm{m})$. The first safety hutch (OpticsHutch) houses the primary slits (PS), several beam diagnostics devices (BPM) and a photon absorber/white beam shutter unit (A/S). The BPM uses pairs of crossed anode wires to detect photo-electrons that are produced by the synchrotron beam in a thin beryllium foil $(50 \mu \mathrm{m})$. This BPM achieves a spatial resolution of $20 \mu \mathrm{m}$ and acts as an on-line intensity monitor and alignment tool for the primary slits. The primary slits select the central cone of the undulator beam and reduce the heatload on the down-

Table 1: Parameters for monochromator crystals. The intrinsic energy resolution is calculated for the symmetrical Bragg case and linear $\sigma$ polarization. The intrinsic resolution for the $(002)$ reflection of a 200 $\mu \mathrm{rad}$ Be mosaic crystal is $5 * 10^{-4}$.

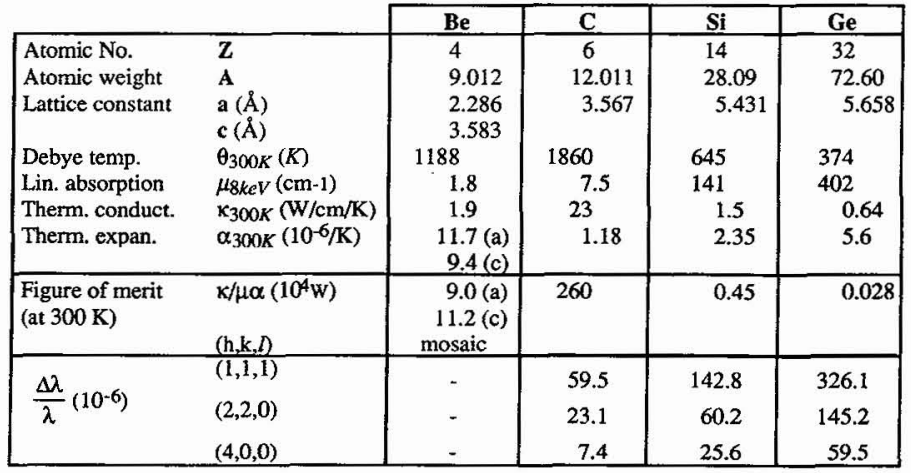




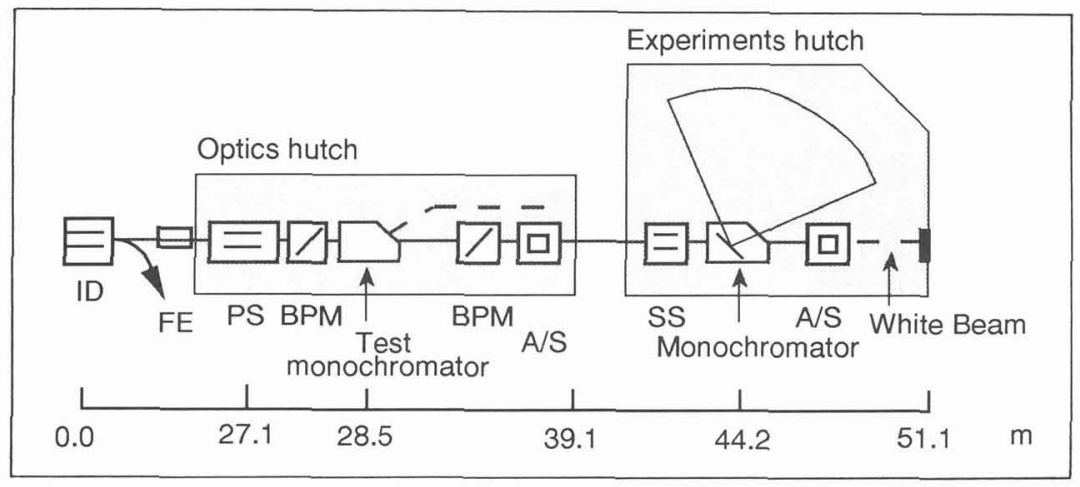

Figure 2: Schematic lay-out of the TROIKA I beamline.

stream optical elements. The Experiments-Hutch contains the monochromator module which is enclosed between two Be-windows $(500 \mu \mathrm{m})$ and comprises a secondary slit unit (SS) and the monochromator unit. The monochromator is located at $44.2 \mathrm{~m}$ from the source and operates with transparent crystals mounted in horizontal Laue geometry. This geometry facilitates to deflect the monochromatic beam out of the horizontal scattering plane [2]. A top view of the monochromator assembly is shown in Figure 3. Pairs of watercooled, chromium plated $\mathrm{Cu}$ cylinders are mounted on small turntables and act as horizontal and vertical slits to control the footprint of the beam on the crystal. The monochromator crystals are attached with a gallium-indium eutectic to a water-cooled $\mathrm{Cu}$-holder. This assembly fits into a goniometer head allowing translation, tilt and rotation of the crystal. The monochromator operates in high-vacuum involving active water-cooling of all motors. A Be window $(500 \mu \mathrm{m})$ for the monochromatic beam allows scattering angles between $20^{\circ}$ and $100^{\circ}$ relative to the forward direction. This aperture defines an energy range between $6 \mathrm{keV}$ and $18 \mathrm{keV}$ (using the diamond (111) reflection). The white transmitted beam is dumped into a photon absorber/beam shutter module (A/S) but may be available for experiments that can be installed in the second half of the Experiments-Hutch. The monochromator module and the PS module are both equipped with three HLS (Hydrostatic Levelling System) sensors [3] that ensure, together with two reference sensors in the storage ring tunnel the mechanical alignment of the beamline. A second transmission monochromator is installed in the Optics-Hutch for test purposes and can be operated simultaneously.

\subsection{The Undulator Source}

The beamline uses a $46 \mathrm{~mm}$ undulator segment (total length $1.6 \mathrm{~m}$, number of periods 36 , $\mathrm{B}_{\max }=0.48 \mathrm{~T}$ ) installed in the ID10 high- $\beta$ straight section. A rms source size of $435 \mu \mathrm{m}$ horizontal, 95 $\mu \mathrm{m}$ vertical and rms divergences of $19 \mu \mathrm{rad}$ horizontal and $13 \mu \mathrm{rad}$ vertical imply that the central cone of the undulator beam at the monochromator ( $44.2 \mathrm{~m}$ from source) has a size (FWHM) of $2.2 \mathrm{~mm}(\mathrm{~h}) * 1.3$ $\mathrm{mm}(\mathrm{v})$. The undulator is built in NdFeB permanent magnet technology and both gap and taper are tunable (20mm minimum gap). The total angle-integrated spectral flux [photons $/ \mathrm{sec} / 0.1 \%$ bandwidth] for the

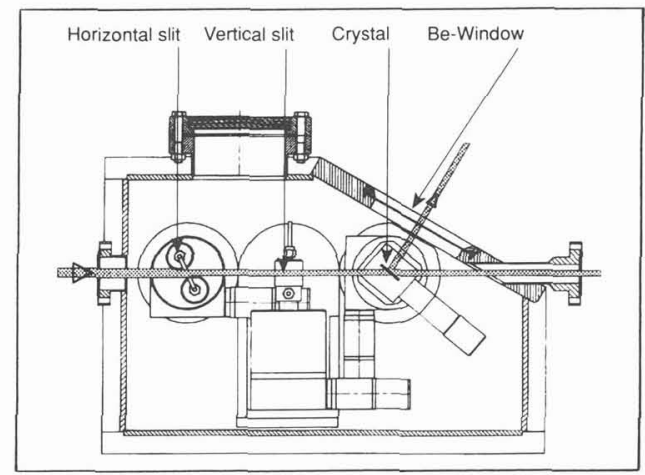

Figure 3: Top view of the monochromator assembly. The length (flange to flange) is $572 \mathrm{~mm}$. 

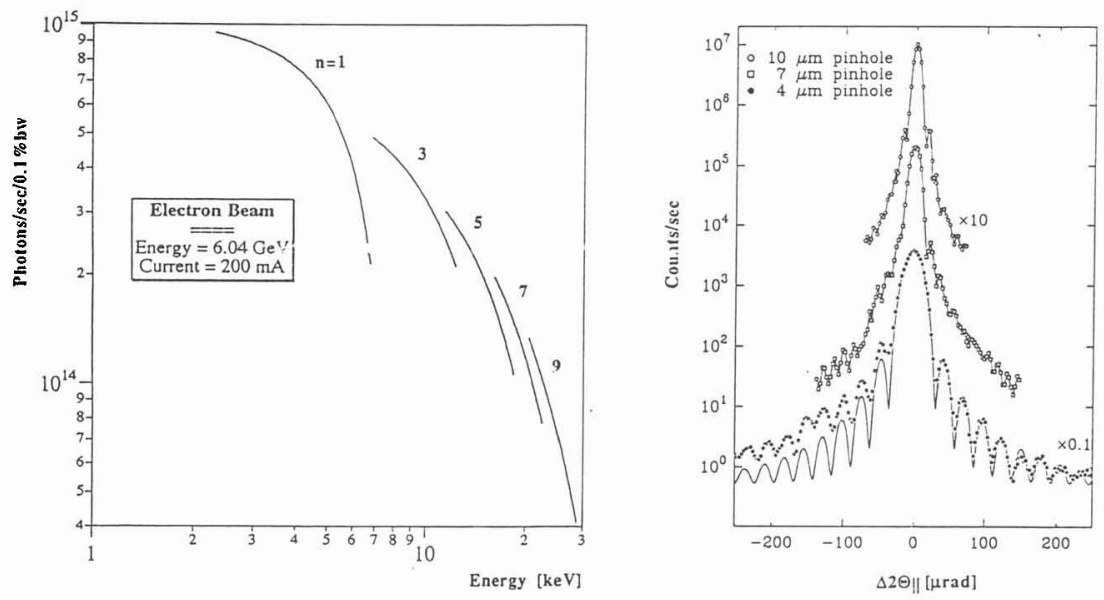

Figure 4: Total angle integrated spectral flux as a function of the photon energy. The data are calculated for $200 \mathrm{~mA}$ ring current and the magnetic gap is varied to tune the harmonics to the required energy. Figure 5: Fraunhofer diffraction patterns from circular pinhole apertures of $4 \mu \mathrm{m}, 7 \mu \mathrm{m}$ and $10 \mu \mathrm{m}$ diameters. The intensity was recorded with a $5 \mu \mathrm{m}$ analyzer pinhole. The solid line (bottom spectrum) represents the calculated diffraction pattern of a circular pinhole convoluted with the resolution of the analyzer pinhole.

odd harmonics 1-9 is shown in Figure 4 as a function of the energy. The power density in the central cone at $30 \mathrm{~m}$ distance and with $\mathrm{K}=2.08$ is $23 \mathrm{Watts} / \mathrm{mm}^{2}$. The total power emitted by the ID is 855 Watts.

The polarization characteristics of the source were measured at $9.1 \mathrm{keV}$ ( $3 \mathrm{rd}$ harmonic) by means of a polarization analyser employing the (331) and (420) reflections of an Al powder. When selecting a small portion of the central cone $\left(2 \times 2 \mu \mathrm{rad}^{2}\right)$ the measured degree of linear polarization is $1.00 \pm 0.02$, while opening the slits (SS) to $10 \times 10 \mu \mathrm{rad}^{2}$ reduces the polarization to $0.97 \pm 0.02$. The coherence properties were studied by measuring Fraunhofer diffraction patterns from circular pinhole apertures [4]. Collimating pinholes of different diameter $(4 \mu \mathrm{m}, 7 \mu \mathrm{m}$ and $10 \mu \mathrm{m})$ located at $45 \mathrm{~m}$ from the source were illuminated by monochromatic $x$-rays $(\lambda=1.05 \AA)$. The narrow settings of the secondary slits in this experiment greatly reduced the heatload and allowed to operate with a $\mathrm{Si}(220)$ monochromator. The primary slits were set to make the horizontal source size viewed by the pinhole equal to the vertical source size, giving a transverse coherence length of $\lambda R_{S} / 2 d_{S} \sim 10 \mu \mathrm{m}$ in both directions, where $R_{S}$ is the distance from the source and $d_{S}$ is the source size.The longitudinal coherence of the beam, $\lambda^{2} / 2 \Delta \lambda$, was $\sim 1 \mu \mathrm{m}$. Figure 5 shows a Fraunhofer pattern recorded by scanning a $5 \mu \mathrm{m}$ analyzer pinhole located at $1.4 \mathrm{~m}$ from the collimating pinhole, parallel to the scattering plane of the monochromator $\Delta 2 \Theta_{\|}$. Comparable Fraunhofer patterns were obtained for the direction perpendicular to the scattering plane of the monochromator. The coherent flux through the $10 \mu \mathrm{m}$ pinhole is $3 \times 10^{7}$ photons/sec.

\subsection{The Transmission Monochromator}

TROIKA I operates without pre-mirror and its operation relies fully on the performance of the transmission-monochromator in the undulator beam. The theoretically expected performance is given in Table 2, summarizing the energy dependence of the energy and angular acceptances of a diamond (111) monochromator in the symmetric Bragg and $35.3^{\circ}$ asymmetric Laue geometries. For both geometries, because of the $\pi$ polarization of the source for a horizontally diffracting monochromator, the energy acceptance is reduced by $\cos 2 \theta$ from the value for $\sigma$ polarization. In addition, the asymmetric Darwin widths are modified by $(\sin \beta / \sin \alpha)^{1 / 2}$, where $\beta$ and $\alpha$ are the angles between the crystal surface and the exit and incident beams, respectively. The emittance angles $\Delta \Theta_{\mathrm{E}}$ for the asymmetric Laue geometry are also given. No degradation due to thermal load is expected according to simple heatload estimates: A beam of radius $R_{1}$ with a power density $P$ impinging normal to the surface on a crystal plate of thickness $t$ and radius $R_{2}$ (cooled on its periphery) yields a temperature rise in the center of $\Delta T \approx R_{1}^{2} * P *(1-\exp (-\mu t)) * \ln \left(R_{2} / R_{1}\right) / 2 t \kappa$. At 8 $\mathrm{keV}$ a $0.3 \mathrm{~mm}$ thick diamond crystal will absorb $20 \%$ of the power. For a power density of $20 \mathrm{Watts} / \mathrm{mm}^{2}$ and $\mathbf{R}_{2}=2 \mathrm{R}_{1} \sim 2 \mathrm{~mm}$ a temperature rise of less than $5 \mathrm{~K}$ is expected. If the same beam is impinging on a Si crystal the temperature would increase by about $150 \mathrm{~K}$. 
Table 2: Energy dependence of the energy and angular acceptances of a diamond (111) crystal in the symmetric Bragg and $35.3^{\circ}$ asymmetric Laue geometries. The values are calculated for a $1000 \mu \mathrm{m}$ thick crystal, for linear $\pi$ polarization of the source and a horizontally-diffracting monochromator. The angular emittance $\Delta \theta_{\mathrm{E}}$ for the asymmetric Laue geometry is also given.

\begin{tabular}{|c|c|c|c|c|c|}
\hline \multirow{2}{*}{$\begin{array}{c}\text { Energy } \\
(\mathrm{keV})\end{array}$} & \multicolumn{2}{|c|}{ Symmetric Bragg } & \multicolumn{3}{|c|}{$35.3^{\circ}$ Asymmetric Laue } \\
\hline & $\begin{array}{c}\Delta \lambda / \lambda \\
\left(10^{-6}\right) \\
\end{array}$ & $\begin{array}{c}\Delta \theta \\
(\mu \mathrm{rad})\end{array}$ & $\begin{array}{l}\Delta \lambda \lambda \\
\left(10^{-6}\right)\end{array}$ & $\begin{array}{c}\Delta \theta \\
(\mu \mathrm{rad})\end{array}$ & $\begin{array}{c}\Delta \theta_{E} \\
(\mu \mathrm{rad})\end{array}$ \\
\hline 6 & 29.6 & 17.1 & 19.1 & 11.1 & 26.5 \\
\hline 8 & 42.7 & 17.3 & 31.7 & 12.9 & 23.3 \\
\hline 9 & 46.2 & 16.4 & 35.7 & 12.7 & 21.2 \\
\hline 10 & 48.7 & 15.4 & 38.8 & 12.3 & 19.3 \\
\hline 12 & 52.0 & 13.5 & 43.2 & 11.2 & 16.2 \\
\hline 14 & 53.9 & 11.9 & 46.2 & 10.2 & 13.9 \\
\hline 16 & 55.3 & 10.6 & 48.2 & 9.2 & 12.1 \\
\hline 18 & 56.2 & 9.5 & 49.8 & 8.5 & 10.8 \\
\hline
\end{tabular}

One natural and several synthetic diamond crystals (typical dimensions: $8 \times 8 \mathrm{~mm}^{2}, 100-500 \mu \mathrm{m}$ thick), cut and polished along the (100) faces have been selected [5] and their performances in the undulator beam (using water-cooling) was studied by taking double-crystal rocking curves with an asymmetric $\mathrm{Si}(220)$ analyser crystal. The convoluted width (FWHM) in this arrangement for two perfect crystals is $22.1 \mu \mathrm{rad}(20.4 \mu \mathrm{rad}$ for the diamond and $4.4 \mu \mathrm{rad}$ for the asymmetric $\mathrm{Si}(220)$ crystal at 9 $\mathrm{keV}$ ). The results for a $202 \mu \mathrm{m}$ thick natural diamond are shown in Figure 6 as a function of the incident beamsize at two different storage ring currents $(8 \mathrm{~mA}$ and $85 \mathrm{~mA})$ in order to demonstrate that the observed broadening does not primarily arise from thermal effects but can be explained by a non-uniform defect distribution within the crystal. The beamsize was increased by successive opening of slits and thereby increasing the illuminated area on the crystal and the incident power as indicated by the (calculated) curves for the incident power. An almost identical rocking curve width (35.6 $\mu \mathrm{rad}$ and 34.1 $\mu \mathrm{rad})$ was observed for drastically different incident powers $(8.3 \mathrm{~W}$ at $8 \mathrm{~mA}$ vs. $85.4 \mathrm{~W}$ at $85 \mathrm{~mA}$, maximum power density: $12 \mathrm{~W} / \mathrm{mm}^{2}$ ) when a beamsize of $12 \mathrm{~mm}^{2}$ was choosen. Identical widths were obtained for two other selected beamsizes $\left(4 \mathrm{~mm}^{2}\right.$ and $\left.0.2 \mathrm{~mm}^{2}\right)$. This behaviour indicates that an inhomogeneous distribution of defects which is successively sampled by increasing the illuminated area causes the broadening of the rocking curves. Rocking curve widths between $24 \mu \mathrm{rad}$ and $45 \mu \mathrm{rad}$ were in fact observed when scanning a very fine beam across this diamond crystal. The existence of such imper-

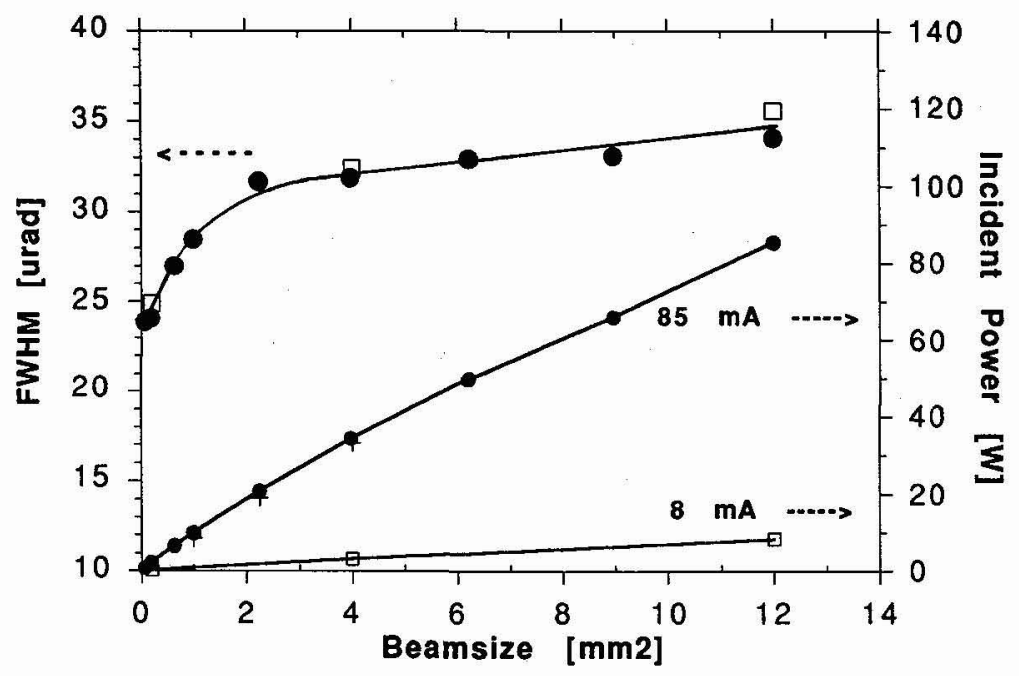

Figure 6: Rocking curve widths of a $202 \mu \mathrm{m}$ thick natural diamond crystal taken with an asymmetric $\mathrm{Si}(220)$ analyser crystal at $9 \mathrm{keV}$ as a function of the incident beam size. The calculated total incident power is given for two storage ring currents. Filled circles (open squares) correspond to width and power for $85 \mathrm{~mA}(8 \mathrm{~mA})$ current. The crossed symbols represent the power as measured with a calorimeter. 


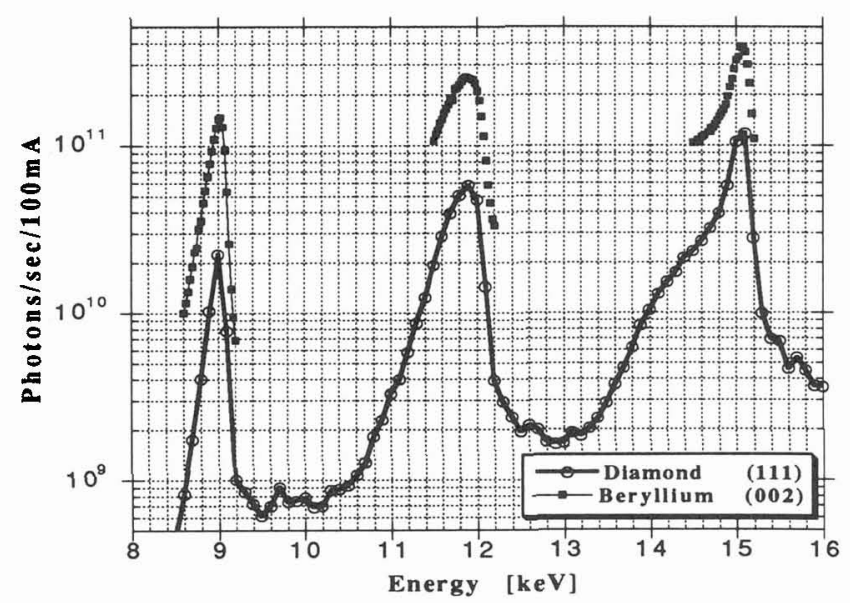

Figure 7: Spectra of the ID10 undulator (harmonics 3,4 and 5) at $21.68 \mathrm{~mm}$ gap recorded with a diamond crystal (open circles) and a mosaic beryllium crystal (solid squares). A $0.5 \times 0.5 \mathrm{~mm}^{2}$ aperture was placed in front of the monochromator (44.2 $\mathrm{m}$ from source).

fections was shown for synthetic diamonds by x-ray topographs [6]. Thermal effects appear to be very small, at least for the power levels encountered at ID10. Only the central part of the undulator beam $\left(1 \mathrm{~mm}^{2}\right)$ is used in standard operation and the rocking width of the diamond under this condition is less than $30 \mu \mathrm{rad}$ and largely independent of the incident power. Slightly broader rocking curves were found for synthetic diamonds. Beryllium mosaic crystals $\left(8 \times 8 \mathrm{~mm}^{2}\right.$, thickness $<1 \mathrm{~mm}$, mosaic distribution typically $200 \mu \mathrm{rad}$ ) were also investigated. No thermal effects were observed in heatload tests. A measure of the spectral flux for the two transparent monochromators discussed was obtained by single photon counting using $90^{\circ}$ scattering from a thin Kapton foil [7] or by using an ionisation chamber. Figure 7 shows the results (harmonic 3,4 and 5) taken at $21.68 \mathrm{~mm}$ gap with a diamond monochromator (open symbols) and a beryllium monochromator (solid squares). A $0.5 \times 0.5 \mathrm{~mm}^{2}$ aperture was placed in front of the monochromator crystals and the measured intensities were normalized to $100 \mathrm{~mA}$ storage ring current. The higher flux achieved with the beryllium monochromator reflects the bigger bandwidth $\left(\Delta \lambda \lambda=5 \times 10^{-4}\right)$ reflected by the mosaic Be crystal.

Focusing and filtering of the beam can be achieved if desireable by introducing flat or bent mirrors in the monochromatic beam. Focusing in the vertical direction was successfully tested with a bent W/Si multilayer ( $2.2 \mathrm{~nm}$ period, radius of curvature: $52.89 \mathrm{~m}$ ). A vertical beamsize of $114 \mu \mathrm{m}$ was achieved.

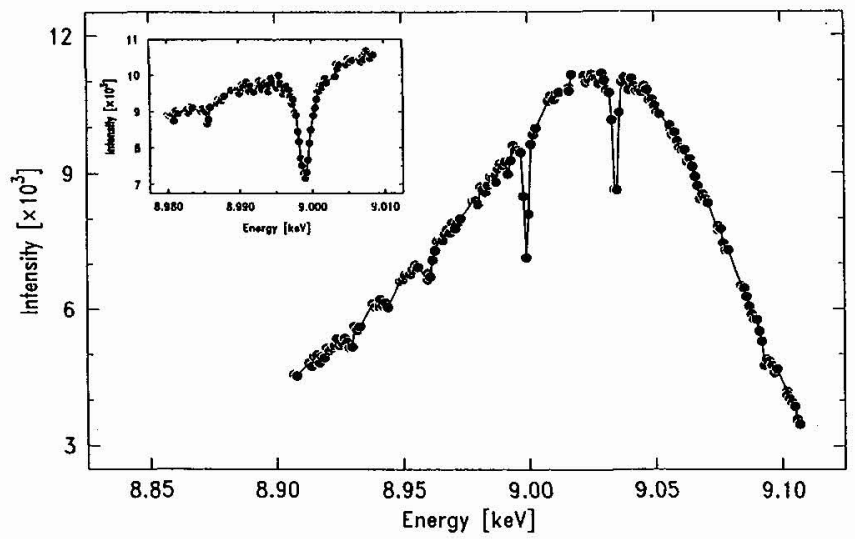

Figure 8: Spectrum of the 3rd undulator harmonic after transmission through a diamond crystal (located $28.5 \mathrm{~m}$ from source), which was aligned to diffract at $8.998 \mathrm{keV}$. The spectrum is taken with a second diamond crystal ( $44.2 \mathrm{~m}$ from source) and exhibits glitches introduced by the first crystal. The insert shows a narrow scan through the main dip with a width of $2 \mathrm{eV}$. 
Discrimination against higher harmonics is obtained with a flat $\mathrm{SiC}$ mirror $\left(\mathrm{E}_{\mathrm{c}}[\mathrm{mrad}]=34 / \mathrm{E}[\mathrm{keV}]\right.$ ). Demagnification in the horizontal plane is intrinsic to the asymmetric Laue geometry. At $9 \mathrm{keV}$ the demagnification $b=\cos (\theta-\alpha) / \cos (\theta+\alpha)=1.67$ with $\theta$ being the Bragg angle and $\alpha(=35.3 \mathrm{deg})$ being the angle between the crystal surface and the normal to the diffracting planes.

A second monochromator (28.5 m from source) can be operated simultaneously to the main monochromator and allows to study the cross-talk between 2 transparent monochromators. For this purpose the diamond crystal in the first monochromator was set to diffract at $E=8.998 \mathrm{keV}$. Figure 8 shows a spectrum of the transmitted beam taken with the downstream monochromator. A series of dips introduced by the first diffracting crystal are clearly visible and have been indexed. The inset shows a narrow scan through the the main dip, corresponding to the (111) reflection of the first diamond crystal. An intensity drop of $30 \%$ and a width of $2 \mathrm{eV}$ were observed for this arrangement of two thin diamond crystals in asymmetric Laue geometry. The quality of the transmitted spectrum illustrates that downstream monochromators can easily be operated in the unperturbed regions of the transmitted spectrum.

\subsection{Instrumentation}

The TROIKA I beamline is equipped with a horizontal diffractometer. Figure 9 shows a side view of this instrument. Two horizontal turntables define the scattering angles and a third turntable can either support pathways for the scattered beam or can carry an additional detector. A vertical translation stage allows measurements in which the sample has to be kept in the horizontal plane. The sample stage can either be equipped with an $x-y$ translation assembly and a double tilt stage with a range of $\pm 20^{\circ}$ or an Eulerian cradle providing full four-circle capability. A flat mirror for harmonic rejection and a two-circle analyser stage for high resolution experiments are part of the diffractometer assembly. The diffractometer is mounted on a platform that can be moved on air-cushions in order to operate at different energies (6 $\mathrm{keV} \leq \mathrm{E} \leq 18 \mathrm{keV}$ ). Reliable operation of the air-pad system is ensured by a leveled epoxy floor that is installed in the monochromator-diffractometer area of the hutch. Movement on a circular path is achieved by connecting the platform to a rotary table mounted underneath the monochromator assembly. The rotary table is mechanically decoupled from the monochromator and alignment between monochromator axis and the rotary table axis is achieved by means of an $x-y$ stage located underneath the rotary table. Energy tuning including movements of the diffractometer and adjustment of the scattering angles (fixed wavevector-transfer mode) is fully computer controlled. All beamline- and diffractometer motions are stepping motor driven. The beamline is controlled via a VME system and supported by a HP 9000-710 workstation. Monochromator and diffractometer are controlled via the SPEC software package interfaced into the VME system.

\section{Commissioning and Operation}

Troika $I$ is a high-brilliance multipurpose undulator beamline and will be fully operational in September 1994. A variety of experiments have already been carried out during the commissioning period in order to explore the performance of the new monochromators and to test the beamline for future

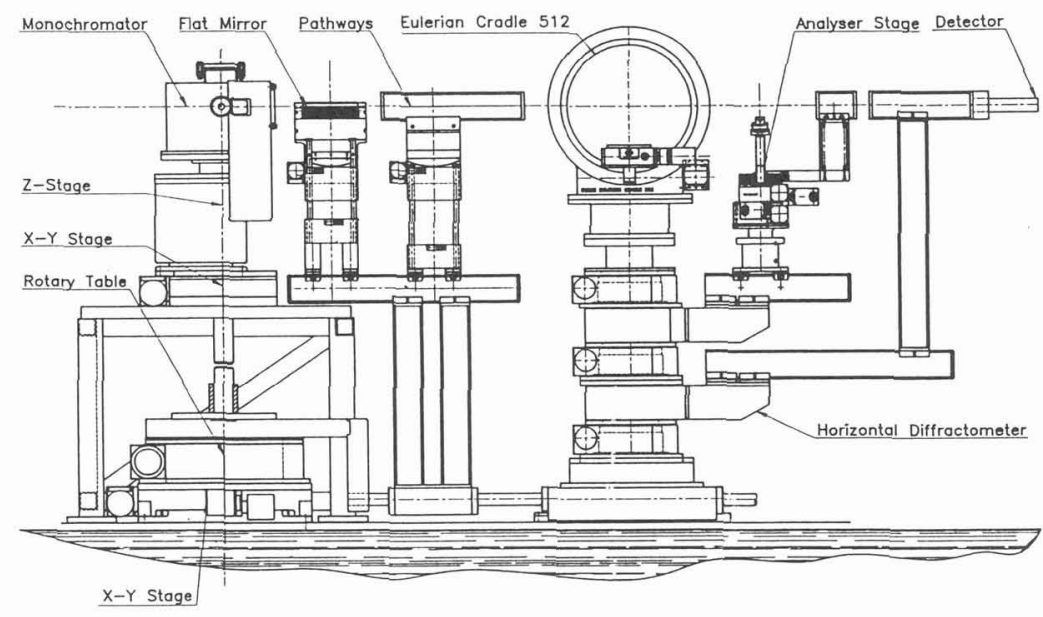

Figure 9: Side view of the TROIKA I horizontal diffractometer. 
applications. These involved studies of the melting of short alcohol monolayers on water and studies of amphiphilic films at the air-liquid interface [8], resonant magnetic scattering in rare-earths and rare-earth compounds [9], protein crystallography in the multiple-wavelength anomalous dispersion and monochromatic mode [10] and scattering with coherent $\mathrm{x}$-rays from multilayers and intensity fluctuation spectroscopy in a binary alloy [11]. The first experiments confirm that beam-multiplexing with transparent monochromators is perfectly feasible and well matched to the characteristics of third generation synchrotron sources. TROIKA I offers energy tunability between 6 and $18 \mathrm{keV}$ and provides one of the most intense coherent $\mathrm{x}$-ray beams available today. Upgrades will involve the exploration of new focussing schemes like Bragg-Fresnel multilayer optics, new detector systems and the installation of additional transparent monochromators to implement the full TROIKA concept.

\section{Acknowledgements}

Research and development on perfect diamond crystals is carried out in collaboration with J.P.F. Sellschop, University of the Witwatersrand, Johannesburg, South Africa. A natural diamond sample was kindly provided by the Avakian Group (Yerewan University) through the courtesy of E. Uggerhøj (Aarhus, Denmark). The support of J.F. Legrand during the commissioning is gratefully acknowledged. The polarization measurements were performed in collaboration with C. Vettier, A. Stunault and F. deBergevin. The focussing studies were performed in collaboration with E. Ziegler and M. Krisch. We thank M. Sanchez del Rio for help in the ray-tracing studies and M. Mattenet for support in the calorimeter tests. The spectral flux spectrum (figure 4) was provided by P. Elleaume. P. Feder, H. Gleyzolle and J. Linderholm have provided valuable technical support.

\section{REFERENCES}

[1] Freund A.K., Joksch St.,Kawata H.,Marot G.,Ziegler E., Berman L.E., and Hastings J.B., Rev. Sci. Instr. 63 (1992) 446-449; Als-Nielsen J. and Freund A. K., Rev. Sci. Instr. 63 (1992) 1156-1159.

[2] Wulff M. and Als-Nielsen J., Rev. Sci. Instr. 63 (1992) 1134-1137.

[3] Roux D., "Particle Accelerator Conference", Washington 17-20 May 1993 (The Institute of Electrical and Electronics Engineering, Piscataway,1993) pp. 2932-2035.

[4] Grübel G., Als-Nielsen J., Abernathy D., Vignaud G., Brauer S., Stephenson G.B., Mochrie S.G.J., Sutton, M., Robinson I.K., Fleming R., Pindak R., Dierker S. and Legrand J.F., ESRF Newsletter 20 (1994) 14-15.

[5] Sellschop J.P.F., Freund, A.K., Als-Nielsen J. and Grübel G., to be published.

[6] Baruchel J. and Zontone F., to be published.

[7] Als-Nielsen J. and Kjaer K., Nucl. Instr. and Meth. A323 (1992) 686-693.

[8] Weinbach S.P., Kjaer K., Grübel G., Legrand J.F., Als-Nielsen J., Lahav M. and Leiserowitz L., to be published; Berge B., Konovalov O., Lajzerowicz J., Renault A., Rieu J.P., Vallade M., AlsNielsen J. and Legrand J.F., submitted for publication

[9] Grübel G., Vettier C., Gibbs D., Bohr J., Als-Nielsen J. and Pengra D., to be published; Stunault A., Vettier C., de Bergevin F., Maier A., Grübel G. and Galera R.M., to be published.

[10] Shapiro L., Hendrickson W.A., Grübel G., Als-Nielsen J., Lehmann M. and Thompson A., to be published; Belrhali H., Yaremchuk A., Tukalo M., Larsen K., Berthet-Colominas C., Leberman R., Beijer B., Sproat B., Als-Nielsen J., Grübel G., Legrand J.F., Lehmann M. and Cusack S., Science 263 (1994) 1432-1436.

[11] Dierker S., Pindak R., Fleming R., Robinson I.K., Grübel G., Als-Nielsen J. and Abernathy D., "Spring Meeting of the American Physical Society", Pittsburgh 21-25 March 1994, p.737;

Brauer S., Stephenson G.B., Sutton M., Bruning R., Dufresne E., Mochrie S.G.J., Grübel G., Als-Nielsen J. and Abernathy D., "Spring Meeting of the American Physical Society", Pittsburgh 21-25 March 1994, p.768. 